\title{
Desarrollo de una Mezcla de Fibras y Almidones como Reemplazante de Grasa para Productos de Pasta Fina tipo Salchicha
}

\author{
Eduar E. Ramírez-Camargo(1), Alejandra M. Marulanda ${ }^{(1)}$ y Jose A. Orrego ${ }^{(2)}$ \\ (1) Tecnas S.A. Cra. 50G No. 12 Sur 29, Itagüí, Colombia, (e-mail: ederaca@yahoo.es; \\ auxinvestigacion2@tecnas.com.co). \\ (2) Universidad de Antioquia, calle 67 No. 53 - 108, Colombia (e-mail: jaorregod@gmail.com)
}

Recibido Mar. 24, 2015; Aceptado Jun. 1, 2015; Versión final Sept. 9, 2015, Publicado Feb. 2016

\begin{abstract}
Resumen
El objetivo de este trabajo fue desarrollar una mezcla de fibras utilizando cáscara de naranja, arveja y almidón de yuca para reemplazar $50 \%$ de grasa en salchichas. Se eliminaron sabores amargos, cítricos en las cascaras; se obtuvo la fibra de arveja y se mezcló con almidón hasta obtener 18 combinaciones. Se evaluó la capacidad de retención de agua y parámetros reométricos (modulo elástico y viscoso) a las mezclas de fibras; parámetros reométricos a la pasta cárnica, análisis de perfil de textura y sensoriales de la salchicha. Las combinaciones de (1.0g naranja. $1.0 \mathrm{~g}$ arveja y $0.25 \mathrm{~g}$ almidón), (1.0g naranja. $1.5 \mathrm{~g}$ arveja y $0.1 \mathrm{~g}$ almidón), (1.38g naranja. $1.25 \mathrm{~g}$ arveja y $0.13 \mathrm{~g}$ almidón), al ser incluidas en la formulación exhibieron comportamientos similares al patrón a nivel sensorial (dureza, cohesividad y masticabilidad) y de textura. Con los resultados obtenidos se concluye que estas combinaciones pueden ser utilizadas para reemplazar la grasa en el producto estudiado.
\end{abstract}

Palabras clave: naranja; arveja; almidón; grasa; salchicha

\section{Development of a Mixture of Fibers and Starches as Fat Replacer for Fine Paste Type Sausage}

\begin{abstract}
The aim of this work was the development of a fiber mixture using orange peel, pea and cassava starch to replace $50 \%$ fat in sausages. Bitter citric flavors were removed from the peels; the pea fiber was obtained and was mixed with starch to get 18 combinations. The water retention capacity and rheometric parameters (elastic and viscous modulus) were evaluated for the mixtures of fibers, rheometric parameters for the meat dough and sensory and profile texture analysis for the sausages. Combinations of $(1.0 \mathrm{~g}$ orange, $1.0 \mathrm{~g}$ pea starch and $0.25 \mathrm{~g})$ ( $1.0 \mathrm{~g}$ orange, $1.5 \mathrm{~g}$ and $0.1 \mathrm{~g}$ pea starch), (1.38g orange, peas and $1.25 \mathrm{~g} 0.13 \mathrm{~g}$ starch), when being included in the formulation exhibited similar behavior pattern for the sensory level (hardness, cohesiveness and chewiness ) and texture. Based on the results it is concluded that these combinations can be used to replace fat in the product studied
\end{abstract}

Keywords: orange; pea; starch; fat; sausage 


\section{INTRODUCCIÓN}

Los consumidores cada vez más se interesan por su salud y demandan de los alimentos que ingieren, además de sabor que sean seguros y saludables. Por esto, la relación entre dieta y salud es más relevante y se buscan alternativas para sentirse bien y permanecer saludables al consumir alimentos funcionales; es decir que estos presenten en su formulación la inclusión de componentes bioactivos a alimentos conocidos que nos los contienen de forma natural, por lo general son de alto consumo (Ospina et al., 2011), y uno de los ingredientes con mayor potencial es la fibra dietaría. La dieta más común en los países desarrollados se caracteriza por un exceso de alimentos muy ricos energéticamente con altos niveles de grasa y azúcares pero con deficiencias de carbohidratos complejos y de constituyentes mayoritarios como la fibra dietaría (Alarcón et al., 2013).

La fibra dietaría ha sido investigada en la ciencia y tecnología de los alimentos debido a su funcionabilidad tecnológica debido a las propiedades que confiere a una matriz alimenticia, de tipo cárnico, lácteo o de galletería; específicamente mejoras en cuanto a la capacidad de adsorción de agua, (CAA), retención de agua (CRA), adsorción de aceite (CAAC) y adsorción de moléculas orgánicas (CAMO) (Alarcón et al., 2013). La fibra dietaría (FD) presente en frutas, vegetales, avena, habas y cereales, es un ingrediente funcional ampliamente usado para desarrollar alimentos nutricionales, debido a los efectos benéficos que presenta en la salud del consumidor. Estas fuentes naturales aportan además pectina, gomas, almidón resistente y otros polisacáridos de reserva que generan soluciones viscosas, facilitando el tránsito intestinal y la adsorción de nutrientes, a su vez reducen el nivel de colesterol en la sangre (Cañas et al., 2011; Verma y Banerjee, 2010). Los compuestos bioactivos y la fibra dietética presentes en residuos agroindustriales de frutas y hortalizas se utilizan ampliamente como ingredientes de alimentos funcionales, debido a sus beneficios potenciales para la salud (Liliana y Cristian, 2014). Sin embargo los residuos industriales de las frutas son una fuente importante de fibra originados dentro de su cadena de transformación. En el procesamiento de naranja para la obtención de zumo se estima que un $50 \%$ es un subproducto, constituido por alvéolo y cáscara; y en Colombia por ejemplo, el desperdicio asciende a 70 mil toneladas por año, generando serios problemas ambientales (Agronet, 2013).

La fibra obtenida de cítricos tiene una gran ventaja frente a las de cereales, porque aporta una mayor cantidad de fibra dietaría soluble, aproximadamente un 33\% comparado con las fibra del salvado de trigo que solo aporta un 7\% (Verma y Banerjee, 2010). Por esta razón se incluye en productos cárnicos, incrementando el rendimiento en el proceso de cocción, debido a las propiedades funcionales como ligazón de agua y grasa, mejorando la textura en embutidos cocidos y madurados (Meneses et al., 2011). Grigelmo-Miguel et al (1999) caracterizaron la fibra obtenida de residuos de naranja y reportaron un contenido de $F D$ total de $35,4 \%$ a $36,9 \%$ en base seca y su potencial uso como espesante, gelificante, texturizante. Las fibras de frutas se han aplicado en salchichas convencionales y reducidas en grasa, se reporta que la luminosidad $\left(L^{*}\right)$ de la salchicha disminuía cuando se incrementaba la concentración de las fibras de naranja o manzana, mientras que con la fibra de durazno fue imperceptible este efecto. Las salchichas reducidas en grasa tienen un menor valor de $L^{*}$, debido a que la grasa aporta características de brillo en el producto, lo cual es una característica deseable en el producto. La cromaticidad amarilla-azul $\left(b^{*}\right)$ se incrementó con la adición de fibra de naranja, además se presentó un olor más alto que el control y una disminución en la jugosidad en el producto final. (García et al, 2007).

El consumo de alimentos con altos contenidos de grasa está asociado con enfermedades cardiovasculares, cáncer y obesidad (Tokusoglu y Ünal, 2003). La reducción de grasa constituye una importante alternativa en la formulación de productos cárnicos saludables; según La Resolución 333 del 2008 del Ministerio de la Protección Social de Colombia, establece que si se elimina el 50\% de las calorías provenientes de la grasa comparado con un alimento de referencia el producto puede considerarse Light. El objetivo de este trabajo fue reducir un $50 \%$ de grasa en un producto emulsionado de pasta fina, tipo salchicha; mediante la inclusión de una mezcla de fibras de naranja, fibra de arveja y almidón de yuca.

\section{MATERIALES Y MÉTODOS}

Materiales: Para la obtención de las mezclas de fibras (MF) y almidones, se procedió obteniendo los residuos de cáscara de naranja en las instalaciones de TECNAS S.A después de realizar la extracción del jugo, los residuos de cáscaras de arveja suministrados por una granja en el oriente antioqueño y el almidón de yuca $(A Y)$ abastecido por una empresa local. Las materias primas constituyentes para la elaboración del embutido se dividieron en dos bloques: el primero; no cárnico, formado por proteína vegetal hidrolizada, condimentos, conservantes, sal, nitral, fosfatos, almidón; y el segundo bloque; el cárnico, conformado por carne de res 80/20 (proteína/grasa), grasa de cerdo, carne mecánicamente deshuesada (CMD) y emulsión de cueros. Las materias primas no cárnicas, sales, fosfatos especias y condimentos fueron suministradas por Tecnas S.A. La carne de res 80/20, emulsión de cueros, (CMD) y grasa de cerdo fueron de consecución 
local; en la Tabla 1, se muestra la composición proximal de las materias primas cárnicas que hicieron parte de la formulación.

Obtención de la fibra de naranja: El proceso de obtención de la fibra de naranja $(F M)$ a partir de los subproductos agroindustriales de naranja (Citrus sinensis) variedad Valencia, fue realizado mediante la aplicación de tratamiento térmico $80^{\circ} \mathrm{C}$ en una marmita marca Talsa con agitación de 15 revoluciones por minuto $(r p m)$, seguido de un filtrado y posterior secado a $60^{\circ} \mathrm{C}$ en un horno Binder FED 115-UL y molienda en un molino Retsch SM 2000 hasta obtener partículas entre $0.149-0.177 \mathrm{~mm}$ (Restrepo et al., 2011). Se realizó un análisis sensorial y bromatológico a la fibra obtenida.

Obtención de la fibra de arveja: Para obtener la fibra de arveja $(F A)$, se realizó secado de la $\mathrm{FA}$ a $60^{\circ} \mathrm{C}$ por 6 horas en un horno Binder FED 115-UL. Seguido de una molienda en un molino Retsch SM 2000 y posterior tamizado para obtener tamaños de partícula entre y $0.149-0.177 \mathrm{~mm}$ (Cañas, 2010).

Tabla 1: Composición proximal de las materias primas cárnicas

\begin{tabular}{|l|c|c|c|c|}
\hline Materia prima & Cenizas (\%) & Grasa total (\%) & Humedad (\%) & Proteína (\%) \\
\hline Carne de res & 1.01 & 0.14 & 78.84 & 20.01 \\
\hline Grasa de cerdo & 0.03 & 88.61 & 8.92 & 2.44 \\
\hline Emulsión de cueros & 1.79 & 16.20 & 72.36 & 9.65 \\
\hline Carne mecánicamente deshusada & 1.15 & 11.82 & 72.04 & 14.99 \\
\hline
\end{tabular}

Análisis Sensorial de la fibra: se realizó con jueces entrenados pertenecientes al panel sensorial de Tecnas S.A. utilizando una escala de 0-7 puntos; en donde se evaluó, sabor amargo, cítrico y sabor naranja, comparándolo con una solución de cafeína al $0.5 \%(\mathrm{w} / \mathrm{w})$ como patrón, bajo la escala Spectrum (Meilgard and Civille 1999).

Análisis Bromatológico de la fibra: la determinación del contenido de cenizas (AOAC 923-03, 2012), proteína (AOAC 988.05, 2012) humedad (AOAC 934.01, 2012) y contenido de fibra soluble (AOAC 993.19, 2012) y fibra insoluble (AOAC 991.42, 2012).

Diseño de mezclas para las fibras: se aplicó un diseño experimental de mezcla $(D O E)$, en el cual se varió el nivel de adición de cada componente, completándose con agua para un total de mezcla de 11,3g (ver Tabla 2) que es la cantidad de grasa de cerdo que se reemplaza dentro de la formulación cárnica. Con las mezclas de fibras (MF) más el agua se garantiza un reemplazo del $50 \%$ de la materia grasa del producto cárnico objeto de estudio. Inicialmente se determinó la capacidad de retención de agua para cada una de las mezclas y luego se procedió a aplicarla en un producto cárnico emulsionado tipo salchicha.

Tabla 2. Diseño de mezclas de fibras y almidones

\begin{tabular}{|c|c|c|c|}
\hline Mezcla & Naranja $(\mathrm{g})$ & Arveja $(\mathrm{g})$ & Almidón $(\mathrm{g})$ \\
\hline 1 & 2,50 & 1,00 & 0,10 \\
\hline 2 & 1,00 & 2,00 & 0,10 \\
\hline 3 & 1,00 & 1,00 & 0,40 \\
\hline 4 & 1,00 & 1,00 & 0,10 \\
\hline 5 & 1,00 & 1,00 & 0,25 \\
\hline 6 & 1,00 & 1,50 & 0,25 \\
\hline 7 & 1,00 & 1,50 & 0,10 \\
\hline 8 & 1,75 & 2,50 & 0,10 \\
\hline 9 & 1,75 & 1,00 & 0,10 \\
\hline 10 & 1,75 & 1,00 & 0,25 \\
\hline 11 & 1,25 & 1,00 & 0,15 \\
\hline 12 & 1,25 & 1,17 & 0,10 \\
\hline 13 & 1,25 & 1,17 & 0,15 \\
\hline 14 & 1,00 & 1,17 & 0,15 \\
\hline 15 & 1,38 & 1,25 & 0,13 \\
\hline 16 & 1,38 & 1,25 & 0,13 \\
\hline 17 & 1,38 & 1,25 & 0,13 \\
\hline 18 & 1,38 & 1,25 & 0,13 \\
\hline
\end{tabular}


Capacidad de retención de agua (CRA): se determinó utilizando una solución salina al $2 \%(\mathrm{w} / \mathrm{w})$ y de acuerdo a la metodología descrita por Delgado y Albarracín, (2012).

Actividad Acuosa: Se determinó utilizando un analizador de actividad de agua Aqualab (DECAGON, serie 3.2) el cual aplica el principio del punto de rocío, en el cual el agua es condensada en la superficie fría del espejo, siendo detectada por un sensor infrarrojo. Las muestras se introducen en la celda a temperatura ambiente y posteriormente en la cámara la cual realiza una medición directa de la actividad de agua.

Microscopía Electrónica de Barrido (SEM): se utilizó un microscopio JEOL JSM 6490 LV, las muestras se fijaron en una cinta de grafito, se les realizó un recubrimiento delgado en oro (Au) (equipo DENTON VACUUM Desk IV) y se analizaron en el microscopio electrónico de barrido en alto vacío con el fin de obtener imágenes en alta resolución. Se empleó el detector de electrones secundarios para evaluar la morfología y topografía de las muestras.

Elaboración del producto cárnico: se pesaron los ingredientes de acuerdo a la formulación indicada en la Tabla 3. Las materias primas cárnicas se molieron a través de un disco de $3 \mathrm{~mm}$ utilizando un molino de carnes EG-22AI Marca Citalsa. Para la elaboración del embutido se utilizó un procesador de alimentos Robocup en donde se adicionaron inicialmente las materias cárnicas junto con los condimentos, la sal, nitral, conservantes y tripolifosfatos, luego se incorporó la proteína vegetal, la mezcla que contiene las MF y una parte de agua. Después se agregó la grasa de cerdo, seguido del conservante, almidón y la otra parte de agua; durante todo el proceso se controló el tiempo de mezclado y la temperatura. Los lotes elaborados fueron de $1,0 \mathrm{Kg}$ de pasta cárnica, con un tiempo total de mezclado para cada lote de 5 minutos, asegurando que la temperatura final de la pasta cárnica fuera menor a $12^{\circ} \mathrm{C}$, para lo cual se adiciono agua-hielo en diferentes momentos del mezclado. La pasta se embutió en fundas de celulosa calibre 22 y se sellaron en ambos extremos. El embutido se sometió a tratamiento térmico en horno Citalsa R-100 hasta alcanzar la temperatura interna de $72^{\circ} \mathrm{C}$, posteriormente se enfrió y se almaceno a $4^{\circ} \mathrm{C}$ para su posterior evaluación.

Tabla 3. Formulación de las salchichas

\begin{tabular}{|l|c|c|}
\hline Ingredientes (\%) & Control & Tratamiento \\
\hline Grasa de cerdo & 11,3 & 5,65 \\
\hline Bloque cárnico & 48,96 & 48,96 \\
\hline Bloque no cárnico & 16,14 & 16,14 \\
\hline Agua-hielo & 23,6 & 23,6 \\
\hline Mezclas de fibras & 0 & 5,65 \\
\hline
\end{tabular}

\section{Caracterización de la pasta cárnica}

pH: se pesó $1,0 \mathrm{~g}$ de la pasta cárnica en $100 \mathrm{~mL}$ de agua destilada y se agito en una placa calefactora CMAG HP 4, pero sin calentamiento por espacio de 5 minutos, luego se procedió a realizar la lectura de pH (Bloukas, Paneras, y Papadima, 1995).

Humedad: se realizó por el método de secado en estufa a $105^{\circ} \mathrm{C}$ hasta peso constante, siguiendo el método de la AOAC 32.1.02 (AOAC 934.01, 2012).

Barrido de temperaturas (medición reológica): se simuló el proceso de cocción de la pasta cárnica, donde se visualizó la gelificación y posterior formación del sólido (salchicha); esto se llevó a cabo utilizando un reómetro Kinexus modelo KNX2100, Malvern Instruments Ltda. Se utilizó la geometría plato-plato dentada de $2 \mathrm{~mm}$ de diámetro y los siguientes parámetros de medición: rampa de temperatura desde $10^{\circ} \mathrm{C}-75^{\circ} \mathrm{C}$ e incrementando la temperatura $2^{\circ} \mathrm{C} /$ minuto, $1 \mathrm{~Hz}$ de frecuencia y $1 \%$ deformación. Se registró la temperatura de gelificación y los valores finales alcanzados en los módulos elástico $\left(G^{\prime}\right)$ y viscoso ( $\left.G^{\prime}\right)$ que alcanzó la pasta cárnica al final del calentamiento $\left(75^{\circ} \mathrm{C}\right)$.

\section{Caracterización del embutido}

Pérdidas por cocción: se registró el peso de las salchichas antes y después del tratamiento térmico y se calculó el porcentaje de pérdida con respecto a la masa inicial (Akesowan, 2008).

Análisis de textura: las muestras a analizar fueron de $25 \mathrm{~mm}$ de longitud, se comprimieron mediante dos superficies planas hasta un $30 \%$ de su altura inicial (compresión del $70 \%$ dos veces sucesivas con el fin de simular la labor que efectuaría la mandíbula humana). Se utilizó un Texturómetro TA-XTplus (Stable Micro Systems) bajo las siguientes condiciones de ensayo: sensor cilíndrico de metal, diámetro de $75 \mathrm{~mm}$ (Sonda 
P75), celda de carga de $25 \mathrm{~kg}$, velocidad de prueba de $4 \mathrm{~mm} / \mathrm{s}$, distancia de penetración de $20 \mathrm{~mm}$. A partir de los perfiles de textura entregados por el equipo se evaluaron los siguientes parámetros: dureza $(N)$, cohesividad (adimensional), elasticidad (adimensional), masticabilidad ( $N s)$ y firmeza $(M)$ (Pons \& Fiszman, 1996).

Análisis sensorial: se realizaron las evaluaciones de acuerdo a la Guía Técnica GTC 165 del 2007. La salchicha fue evaluada mediante pruebas descriptivas, siguiendo los lineamientos descritos en la Norma Técnica Colombiana NTC 4489 de 1998, utilizando 7 jueces entrenados pertenecientes a Tecnas S.A., según la Norma Técnica Colombiana NTC 4129 de 1998. Para la ejecución de la prueba sensorial, previamente fueron escogidos los siguientes descriptores sensoriales: elasticidad, jugosidad, dureza, cohesividad, masticabilidad y sabor característico y no característico, los cuales fueron calificados en una escala de intensidad de 0 - 7 puntos, donde: 0 (ausente), 1 y 2 (leve), 3 (media-baja), 4 (media), 5-6 (mediaalta), y 7 (intenso). Las pruebas fueron realizadas por triplicado bajo condiciones controladas de temperatura y luz. Los resultados se analizaron con el Statgraphis Centurion ${ }^{\circledR} \mathrm{XV}$, con un nivel de confianza del $95 \%$.

Humedad: se realizó por el método de secado en estufa a $105^{\circ} \mathrm{C}$ hasta peso constante, siguiendo lo descrito por la AOAC 32.1.02 (AOAC 934.01, 2012).

\section{RESULTADOS Y DISCUSIÓN}

\section{Obtención de la fibra}

Los sabores amargos y cítricos en el proceso de obtención de la fibra de naranja fueron disminuidos satisfactoriamente, de tal manera que pudo ser incorporada en niveles hasta del $2,5 \%$ en el producto cárnico; el protocolo utilizado permitió eliminar los compuestos que le confieren a la corteza el gusto amargo como algunos bioflavonoides, aceites esenciales; así como la solubilización en las aguas de lavado de la naringina y hesperidina, siendo estos compuestos los responsables de conferir sabores amargos y cítricos (Restrepo et al.,, 2011), en la Figura 1, se muestra la evaluación sensorial realizada para dos tratamientos seleccionados para la obtención de la $F N$; donde el tratamiento 12 reduce en un $65 \%$ la percepción del sabor cítrico.

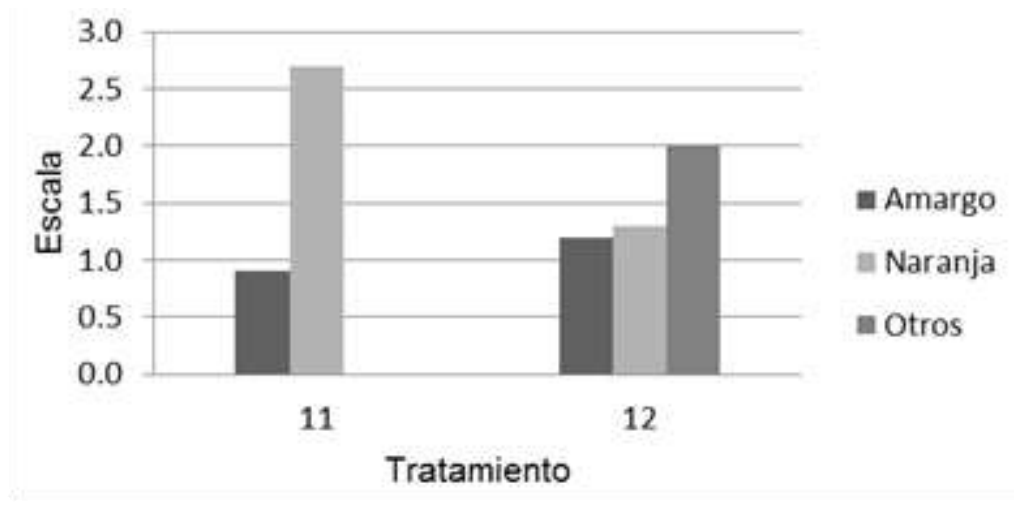

Fig. 1: Evaluación sensorial de la fibra de naranja.

El análisis bromatológico de la fibra de naranja reportó un contenido de fibra total del $71,15 \% ; 4,54 \%$ proteínas, $1,69 \%$ grasa y $3,97 \%$ cenizas; calculados en base seca y concuerdan con el estudio realizado por Simplicio de Santana (2005) quien evaluó la fibra de naranja y maracuyá reportando un valor de fibra del $76,50 \% ; 8,95 \%$ proteína, $2,82 \%$ grasa y $3,90 \%$ cenizas para la naranja.

Al obtener la fibra se debe realizar su posterior caracterización fisicoquímica y funcional en donde la capacidad de retención de agua juega un papel fundamental en la formulación del producto cárnico. Las diferentes mezclas realizadas con la fibra de naranja presentaron un valor de actividad de agua de $0,4723 \pm$ 0,0176, una distribución de tamaño de partícula $D[4,3]$ de $146.2 \pm 32,23(\mu \mathrm{m})$ y $D[3,2]$ de $59,2 \pm 7,8(\mu \mathrm{m})$ y un pH en solución de 6,28 $\pm 0,25$; En la tabla 4, se muestran los resultados encontrados en la determinación de CRA de la fibra y la caracterización de la pasta cárnica. El pH de la pasta cárnica adicionada con las diferentes mezclas estuvo comprendido entre 6,06 y 6,47 , que fue menor con respecto a la muestra control ( $\mathrm{pH}$ de 6,9) esta leve disminución de $\mathrm{pH}$ se puede atribuir a la inclusión de fibra de naranja cuyo $\mathrm{pH}$ en solución al $1 \%$ oscilo entre 5,69 y 6,63. 
Tabla 4. Resultados de la determinación de la capacidad de retención de agua para la fibra y caracterización fisicoquímica y reológica de la pasta cárnica. Muestras con diferentes letras presentan diferencias estadísticamente significativas

\begin{tabular}{|c|c|c|c|c|}
\hline \multirow[b]{2}{*}{ Muestra } & \multirow{2}{*}{$\begin{array}{c}\text { Fibras } \\
\text { CRA }\end{array}$} & \multicolumn{3}{|c|}{ Pasta cárnica } \\
\hline & & Humedad \% & $\mathrm{G}^{\prime}(\mathrm{Pa})$ & $\mathrm{G}^{\prime \prime}(\mathrm{Pa})$ \\
\hline 1 & $9,0 \pm 1,2^{\mathrm{abc}}$ & $68,9 \pm 0,9^{b c}$ & $7,98 \mathrm{E}+04 \pm 8,9 \mathrm{E}+03^{\mathrm{a}}$ & $1,19 E+04 \pm 2,5 E+03^{a}$ \\
\hline 15 & $8,9 \pm 1,2^{\mathrm{abc}}$ & $68,9 \pm 0,9 \mathrm{bc}$ & $7,93 E+04 \pm 8,9 E+03^{a}$ & $1,49 \mathrm{E}+04 \pm 2,5 \mathrm{E}+03^{\mathrm{ab}}$ \\
\hline 9 & $28,1 \pm 1,2^{g h}$ & $68,6 \pm 0,9 \mathrm{bc}$ & $8,28 \mathrm{E}+04 \pm 8,9 \mathrm{E}+03^{\mathrm{a}}$ & $1,68 \mathrm{E}+04 \pm 2,5 \mathrm{E}+03^{\mathrm{ab}}$ \\
\hline 12 & $28,1 \pm 1,2^{g h}$ & $69,9 \pm 0,9^{c}$ & $8,40 \mathrm{E}+04 \pm 8,9 \mathrm{E}+03^{\mathrm{a}}$ & $1,71 \mathrm{E}+04 \pm 2,5 \mathrm{E}+03^{\mathrm{abc}}$ \\
\hline 10 & $24,4 \pm 1,2^{g}$ & $68,8 \pm 0,9 b c$ & $8,60 \mathrm{E}+04 \pm 8,9 \mathrm{E}+03^{\mathrm{a}}$ & $1,60 \mathrm{E}+04 \pm 2,5 \mathrm{E}+03^{\mathrm{ab}}$ \\
\hline 8 & $13,2 \pm 1,2^{\text {def }}$ & $68,6 \pm 0,9 \mathrm{bc}$ & $8,11 \mathrm{E}+04 \pm 8,9 \mathrm{E}+03^{\mathrm{a}}$ & $1,59 E+04 \pm 2,5 E+03^{a b}$ \\
\hline 14 & $7,5 \pm 1,2^{\mathrm{ab}}$ & $67,6 \pm 0,9 \mathrm{bc}$ & $8,86 E+04 \pm 8,9 E+03^{a}$ & $1,49 E+04 \pm 2,5 E+03^{a b}$ \\
\hline 11 & $10,3 \pm 1,2^{\mathrm{abcd}}$ & $67,9 \pm 0,9 \mathrm{bc}$ & $8,85 \mathrm{E}+04 \pm 8,9 \mathrm{E}+03^{\mathrm{a}}$ & $1,73 \mathrm{E}+04 \pm 2,5 \mathrm{E}+03^{\mathrm{abc}}$ \\
\hline 17 & $11,4 \pm 1,2^{\text {cde }}$ & $68,9 \pm 0,9 b c$ & $1,18 \mathrm{E}+05 \pm 8,9 \mathrm{E}+03^{\mathrm{bc}}$ & $1,69 \mathrm{E}+04 \pm 2,5 \mathrm{E}+03^{\mathrm{ab}}$ \\
\hline 6 & $15,5 \pm 1,2^{f}$ & $69,0 \pm 1,3 \mathrm{bc}$ & $1,18 \mathrm{E}+05 \pm 8,9 \mathrm{E}+03^{\mathrm{bc}}$ & $1,68 \mathrm{E}+04 \pm 2,5 \mathrm{E}+03^{\mathrm{ab}}$ \\
\hline 13 & $10,8 \pm 1,2^{\text {bcde }}$ & $68,7 \pm 1,3 \mathrm{bc}$ & $1,21 \mathrm{E}+05 \pm 8,9 \mathrm{E}+03^{\mathrm{bc}}$ & $2,00 \mathrm{E}+04 \pm 2,5 \mathrm{E}+03^{\mathrm{bcd}}$ \\
\hline 3 & $7,7 \pm 1,2^{\mathrm{ab}}$ & $67,5 \pm 1,3 \mathrm{bc}$ & $1,25 \mathrm{E}+05 \pm 8,9 \mathrm{E}+03^{\mathrm{bcd}}$ & $1,97 \mathrm{E}+04 \pm 2,5 \mathrm{E}+03^{\mathrm{bcd}}$ \\
\hline 4 & $11,4 \pm 1,2^{\text {cde }}$ & $69,0 \pm 1,3 \mathrm{bc}$ & $1,00 \mathrm{E}+05 \pm 8,9 \mathrm{E}+03^{\mathrm{ab}}$ & $2,02 \mathrm{E}+04 \pm 2,5 \mathrm{E}+03^{\mathrm{bcd}}$ \\
\hline 16 & $10,2 \pm 1,2^{\mathrm{abcd}}$ & $69,8 \pm 0,9^{c}$ & $1,49 \mathrm{E}+05 \pm 8,9 \mathrm{E}+03^{\mathrm{d}}$ & $3,42 E+04 \pm 2,5 E+03^{f}$ \\
\hline 7 & $14,1 \pm 1,2^{\text {ef }}$ & $69,1 \pm 0,9 \mathrm{bc}$ & $1,15 \mathrm{E}+05 \pm 8,9 \mathrm{E}+03^{\mathrm{bc}}$ & $1,51 \mathrm{E}+04 \pm 2,5 \mathrm{E}+03^{\mathrm{ab}}$ \\
\hline 5 & $6,8 \pm 1,2^{\mathrm{a}}$ & $66,8 \pm 0,9^{b}$ & $1,35 \mathrm{E}+05 \pm 8,9 \mathrm{E}+03^{\mathrm{cd}}$ & $2,45 \mathrm{E}+04 \pm 2,5 \mathrm{E}+03^{\mathrm{cde}}$ \\
\hline 18 & $6,8 \pm 1,2^{a}$ & $68,9 \pm 0,9 \mathrm{bc}$ & $1,38 \mathrm{E}+05 \pm 8,9 \mathrm{E}+03^{\mathrm{cd}}$ & $2,78 \mathrm{E}+04 \pm 2,5 \mathrm{E}+03^{\mathrm{ef}}$ \\
\hline 2 & $8,6 \pm 1,2^{\mathrm{abc}}$ & $68,6 \pm 0,9$ bc & $1,39 \mathrm{E}+05 \pm 8,9 \mathrm{E}+03^{\mathrm{cd}}$ & $2,63 E+04 \pm 2,5 E+03^{\text {de }}$ \\
\hline Control & ----- & $61,3 \pm 0,6^{a}$ & $8.09 E+04 \pm 5,2 E+03^{a}$ & $1,49 E+04 \pm 1,4 E+03^{a b}$ \\
\hline
\end{tabular}

Las mezclas evaluadas presentaron diferencias estadísticas significativas $(p<0.05)$ en términos de su CRA; la cual vario entre $6,8 \pm 1,2$ y $28,1 \pm 1,2$; donde las mezclas 9,10 y 12 presentaron los mayores valores; mientras que las mezclas 5 y 18 exhibieron un menor valor, como se aprecia en la Fig. 2; estas diferencias en CRA están asociadas al comportamiento fisicoquímico de cada uno de los componentes de las MF de manera individual y específicamente a la fibra de naranja obtenida. La fibra ha sido muy investigada en la ciencia y tecnología de los alimentos por las propiedades tecnológicas que estas confieren, las cuales dependen de su composición química, la presencia de poros y su microestructura que afectan la capacidad de absorber y retener agua, formación de gel y la viscosidad (Alarcón et al., 2013).

En la Fig. 3, se observan Microscopías Electrónicas de Barrido (SEM), para las mezclas seleccionadas 5, 7 y 17; de igual forma para las materias primas en forma individual. Las micrografías de los materiales por separado muestran diferentes morfologías, para el $A Y$ (Fig. $3 b$ ) se ven estructuras pequeñas y redondeadas, mientras que las fibras son estructuras con un mayor tamaño y de forma laminar, además se observa que la $F A$ (Fig. 3c) tiene espacios intersticiales mientras que la $F N$ (Fig. 3a) presenta una estructura más compacta. Al ver las micrografías de las MF, se observa una menor presencia de fibras en la mezcla 5 (Fig. 3d), lo cual esta correlacionado con la composición de la mezcla, la cual tiene un $2 \%(\mathrm{w} / \mathrm{w})$ de fibras y un $0.25 \%(\mathrm{w} / \mathrm{w}) A Y$, mientras que las mezclas 7 y 17 tienen un $2,5 \%(\mathrm{w} / \mathrm{w})$ de fibras y solo un $0,1 \%(\mathrm{w} / \mathrm{w}) A Y$, esto hace que la Capacidad de retención de agua de las ultimas mezclas sea mucho mayor comparada con la mezcla 5.

Para altos valores de CRA se observa una correlación entre esta variable y los parámetros reométricos, así una mayor $C R A$ corresponde a un menor valor del módulo elástico como se determinó para las mezclas 9 , 10 y 12 , las cuales no presentaron diferencia estadísticamente significativa $(p>0.05)$ tanto en la $C R A$ como en el valor de $\boldsymbol{G}^{\prime}\left(8,43 \times 10^{4} \mathrm{~Pa}\right)$, hecho explicable por una mayor cantidad de agua que afecta la estructuración del sistema. El otro grupo no presentó correlación entre estas dos variables atribuido a la composición inherente de la mezcla. La pasta cárnica elaborada con fibras presentó un mayor valor de humedad $(68,5 \%)$ con respecto al patrón $(61 \%)$, esto se da por una mayor adición de agua dentro de la formulación en las pastas cárnicas con reducción de grasa, la cual es compensada por la inclusión de la fibra y su correspondiente agua de hidratación. 


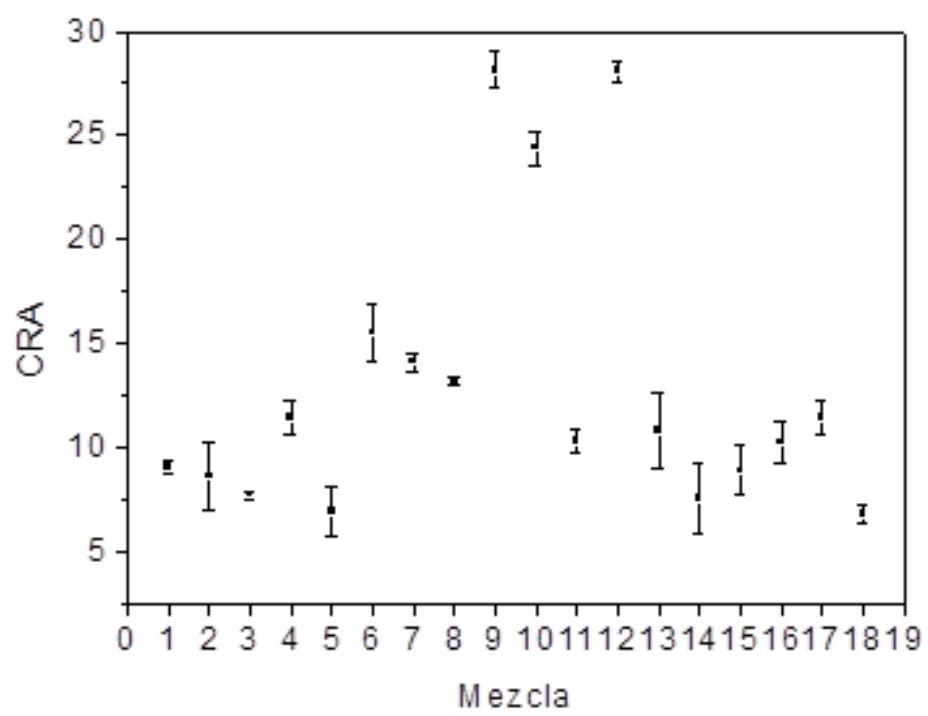

Fig. 2: Capacidad de retención de agua para las diferentes mezclas que contienen fibra de naranja.
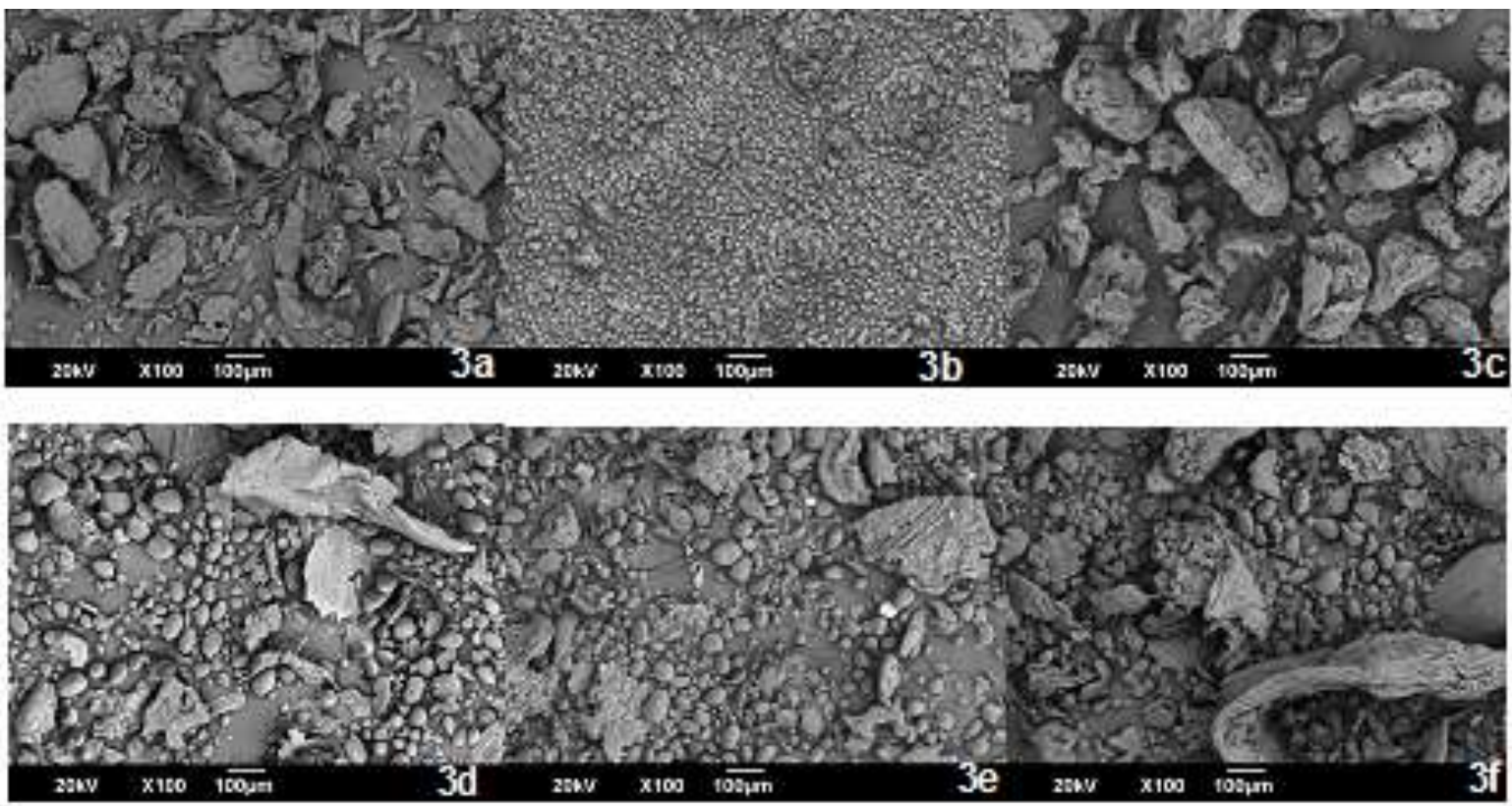

Fig. 3: SEM para los materiales individuales y las mezclas: 3a) FN, 3b) AY, 3c) FA, 3d) MF 5, 3e) MF 7, $3 f)$ MF 17.

Al realizar el barrido de temperatura para la pasta cárnica se registró el valor final alcanzado para los módulos G' y G" de las diferentes mezclas lográndose apreciar 3 grupos homogéneos; el primero conformado por las mezclas: $1,8,9,10,11,12,14$ y 15 las cuales no presentaron diferencias estadísticamente significativas frente a la muestra control, con un valor promedio de $G^{\prime}$ de $8,85 \times 10^{4} \pm$ $3,67 \times 10^{3} \mathrm{~Pa}$, frente al valor de la muestra control que fue de $8,09 \times 10^{4} \pm 5,2 \times 10^{3}$, los otros dos grupos presentan una mayor estructuración y están conformados por las muestras 3, 4, 6, 7, 13 y 17 ( $\boldsymbol{G}^{\text {' }}$ $1,16 \times 10^{5} \pm 8,6 \times 10^{3} \mathrm{~Pa}$ ) y para las muestras $2,5,16$ y $18\left(\boldsymbol{G}^{\prime} 1,40 \times 10^{5} \pm 6 \times 10^{3} \mathrm{~Pa}\right)$, además de presentar estas diferencias en el módulo elástico también se logran encontrar diferentes temperaturas de gelificación, como se observa en la Figura 4.

Estas propiedades viscoelásticas de las $M F$, se atribuye a la $F N$ por presentar conjuntamente propiedades de flujo viscoso y sólido elástico, factores tales como la presión de turgencia, rigidez de la pared celular, contenido de fibra y agua, tiempo, temperatura y dimensiones geométricas del producto determinan el comportamiento reológico de esta en la matriz aplicada (Restrepo et al., 2011). 


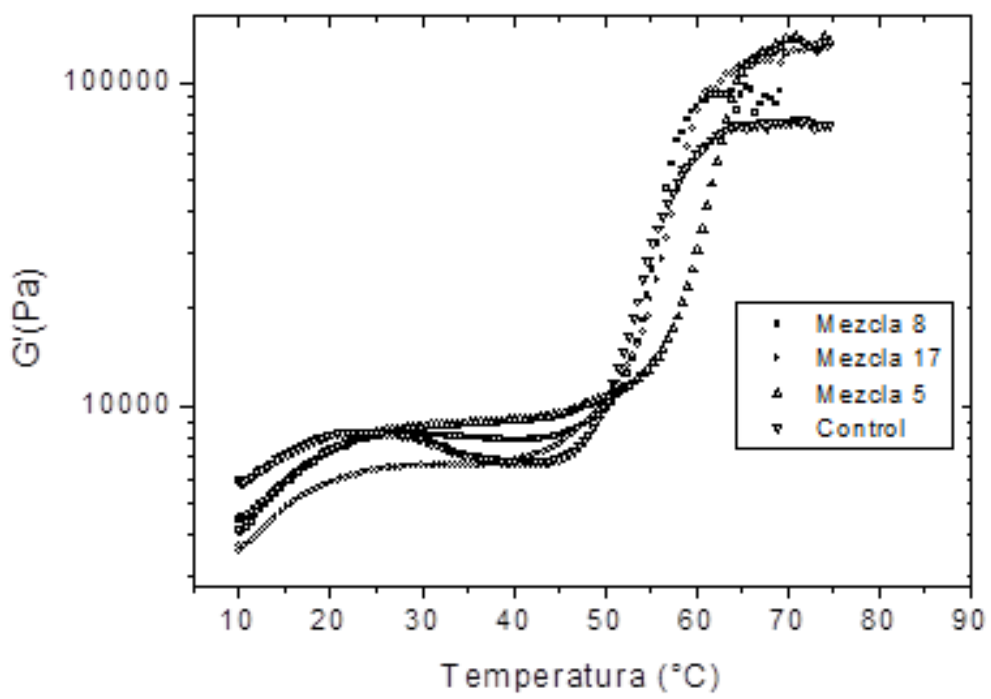

Fig. 4: Barrido de temperaturas para tres mezclas representativas de los grupos homogéneos y la muestra control Convenciones: rojo (G'), azul $\left(G^{\prime}\right)$ y verde $(\delta)$.

El patrón presentó una menor estructuración que las muestras que contienen fibras (excepto para las mezclas 15 y 1), lo cual se ve reflejado en los valores de los módulos de $G^{\prime} 8.09 \times 10^{4} \mathrm{~Pa}$ y G" $1.49 \times 10^{4} \mathrm{~Pa}$, para el patrón y las mezclas oscilaron entre $G^{\prime}: 7,49 \times 10^{4}-1.5 \times 10^{5} \mathrm{~Pa}$ y $\mathrm{G}^{\prime \prime}: 1.2 \times 10^{4}-3.4 \times 10^{4} \mathrm{~Pa}$, este incremento en las propiedades viscoelásticas se da por la presencia de las fibras. La mezcla 16 presentó una mayor estructuración que las otras formulaciones, atribuido a una mayor cantidad de almidón, mientras que los otros componentes ( $F N$ y $F A$ ) están en el nivel medio. La mezcla 1 presentó el valor mínimo de los módulos, esta contiene niveles bajos de $F A$ y $A Y$. En la Tabla 5, se muestran los resultados de la caracterización fisicoquímica y reológica del producto cárnico.

Tabla 5. Propiedades fisicoquímicas y reológicas del producto cárnico. Muestras con diferentes letras presentan diferencias estadísticamente significativas.

\begin{tabular}{|c|c|c|c|c|}
\hline Muestra & $\begin{array}{l}\text { Pérdidas por cocción del } \\
\text { embutido cárnico (\%) }\end{array}$ & Humedad (\%) & $\mathrm{G}^{\prime}(\mathrm{Pa})$ & G" (Pa) \\
\hline 1 & 6.34 & $61,0 \pm 1,0^{\mathrm{bc}}$ & $3,57 E+04 \pm 5,0 E+03^{f}$ & $9,15 E+03 \pm 9 E+02^{f}$ \\
\hline 15 & 6,53 & $63,6 \pm 1,0^{\text {cd }}$ & $2,82 \mathrm{E}+04 \pm 5,0 \mathrm{E}+03^{\text {def }}$ & $7,28 \mathrm{E}+03 \pm 9 \mathrm{E}+02^{\mathrm{efg}}$ \\
\hline 9 & 7,83 & $62,2 \pm 1,0^{\text {bcd }}$ & $7,33 \mathrm{E}+03 \pm 5,0 \mathrm{E}+03^{\mathrm{a}}$ & $2,93 \mathrm{E}+03 \pm 9 \mathrm{E}+02^{\mathrm{a}}$ \\
\hline 12 & 6,67 & $64,5 \pm 1,0^{\mathrm{d}}$ & $1,30 \mathrm{E}+04 \pm 5,0 \mathrm{E}+03^{\mathrm{abc}}$ & $3,95 \mathrm{E}+03 \pm 9 \mathrm{E}+02^{\mathrm{abc}}$ \\
\hline 10 & 6,15 & $61,9 \pm 1,0^{\text {bcd }}$ & $1,06 \mathrm{E}+04 \pm 5,0 \mathrm{E}+03^{\mathrm{abc}}$ & $4,27 E+03 \pm 9 E+02^{a b c d}$ \\
\hline 8 & 5,43 & $63,7 \pm 1,0^{\text {cd }}$ & $1,14 \mathrm{E}+04 \pm 5,0 \mathrm{E}+03^{\mathrm{abc}}$ & $4,38 E+03 \pm 9 E+02^{a b c d}$ \\
\hline 14 & 5,75 & $59,4 \pm 1,0^{b}$ & $8,18 \mathrm{E}+03 \pm 5,0 \mathrm{E}+03^{\mathrm{ab}}$ & $2,94 \mathrm{E}+03 \pm 9 \mathrm{E}+02^{\mathrm{a}}$ \\
\hline 11 & 5,69 & $63,7 \pm 1,0^{\text {cd }}$ & $9,98 \mathrm{E}+03 \pm 5,0 \mathrm{E}+03^{\mathrm{ab}}$ & $3,51 \mathrm{E}+03 \pm 9 \mathrm{E}+02^{\mathrm{a}}$ \\
\hline 17 & 6,33 & $63,1 \pm 1,0^{\text {cd }}$ & $2,55 \mathrm{E}+04 \pm 5,0 \mathrm{E}+03^{\text {cdef }}$ & $6,28 \mathrm{E}+03 \pm 9 \mathrm{E}+02^{\text {bcdef }}$ \\
\hline 6 & 6,49 & $62,9 \pm 1,0^{\text {cd }}$ & $1,95 \mathrm{E}+04 \pm 5,0 \mathrm{E}+03^{\text {abcde }}$ & $5,25 E+03 \pm 9 E+02^{a b c d e}$ \\
\hline 13 & 6,93 & $61,2 \pm 1,0^{\text {bc }}$ & $1,82 \mathrm{E}+04 \pm 5,0 \mathrm{E}+03^{\mathrm{abcd}}$ & $4,96 \mathrm{E}+03 \pm 9 \mathrm{E}+02^{\mathrm{abcde}}$ \\
\hline 3 & 7,10 & $61,1 \pm 1,0^{\mathrm{bc}}$ & $1,22 \mathrm{E}+04 \pm 5,0 \mathrm{E}+03^{\mathrm{abc}}$ & $3,73 \mathrm{E}+03 \pm 9 \mathrm{E}+02^{\mathrm{bc}}$ \\
\hline 4 & 6,91 & $64,5 \pm 1,0^{d}$ & $2,54 \mathrm{E}+04 \pm 5,0 \mathrm{E}+03^{\text {cdef }}$ & $6,52 E+03 \pm 9 E+02^{\text {cdefg }}$ \\
\hline 16 & 6,8 & $63,0 \pm 1,0 \mathrm{~cd}$ & $2,29 \mathrm{E}+04 \pm 5,0 \mathrm{E}+03^{\text {bcdef }}$ & $6,78 \mathrm{E}+03 \pm 9 \mathrm{E}+02^{\text {defg }}$ \\
\hline 7 & 7,57 & $62,7 \pm 1,0^{\text {cd }}$ & $2,03 \mathrm{E}+04 \pm 5,0 \mathrm{E}+03^{\text {abcde }}$ & $5,32 \mathrm{E}+03 \pm 9 \mathrm{E}+02^{\mathrm{abcde}}$ \\
\hline 5 & 6,07 & $64,8 \pm 1,0^{\mathrm{d}}$ & $8,81 \mathrm{E}+03 \pm 5,0 \mathrm{E}+03^{\mathrm{ab}}$ & $2,94 \mathrm{E}+03 \pm 9 \mathrm{E}+02^{\mathrm{a}}$ \\
\hline 18 & 7,17 & $61,9 \pm 1,0^{\text {bcd }}$ & $3,19 \mathrm{E}+04 \pm 5,0 \mathrm{E}+03^{\text {def }}$ & $7,99 \mathrm{E}+03 \pm 9 \mathrm{E}+02^{\mathrm{fg}}$ \\
\hline 2 & 7,10 & $63,2 \pm 1,0^{\text {cd }}$ & $3,31 E+04 \pm 5,0 E+03^{\text {ef }}$ & $7,44 \mathrm{E}+03 \pm 9 \mathrm{E}+02^{\text {efg }}$ \\
\hline Control & 5,34 & $47,5 \pm 0,6^{a}$ & $2,67 \mathrm{E}+04 \pm 3,5 \mathrm{E}+03^{\text {def }}$ & $6,72 \mathrm{E}+03 \pm 96+02^{\mathrm{ef}}$ \\
\hline
\end{tabular}

Las muestras con inclusión de mezclas de fibras y almidones presentaron un leve incremento en las pérdidas por cocción comparadas con el patrón, estas oscilaron entre 5,43 - 7,83\%, siendo la mezcla 8 la que reporto una pérdida similar al patrón 5,34\% y la mezcla 9 la de mayor pérdida por cocción 7.83\%, esta se puede atribuir a una mayor $C R A$ de esta mezcla cuyo valor fue de 1:28 (agua:fibra), mientras que para la mezcla 8 su CRA fue de 1:13. 
El análisis sensorial realizado a las aplicaciones de las $M F$ en el producto cárnico presentaron diferencias estadísticas significativas en el sabor característico entre el patrón y las mezclas 1, 2, 4, 9, 12,13,15, con un valor $p<0,05$, el patrón presentó una calificación promedio de 5,02 mientras que las muestras 9 y 15 fueron las que obtuvieron una menor calificación de 3,78 y 4,06 respectivamente, evaluados en una escala de 0 7; para la variable sabor extraño no se presentaron diferencias estadísticas significativas con un valor $p>0,05$, sin embargo la mezclas 4,10 y 11 presentaron valores más altos, alrededor de 0,86 con respecto al patrón cuyo valor fue de 0,65 en esta característica según la escala evaluada.

Para la variable jugosidad, se presentaron diferencias estadísticas significativas $p<0,05$ entre el patrón y los distintos tratamientos, encontrándose que las muestras $10,11,12,14,16$ recibieron una calificación diferente al patrón, ubicándose en dos regiones: baja jugosidad<2,08 para las muestra 10,11, 14 y 16, alta jugosidad para la muestra 12 con un valor de 3,26; mientras que el patrón tuvo un valor de 2,64. Dentro de las formulaciones alimentarias la grasa contribuye a la jugosidad tanto de productos cárnicos como de los productos lácteos y otros sistemas alimentarios. La jugosidad en la carne y los derivados cárnicos, está asociada a la grasa, por su efecto estimulador en la producción de saliva; más que el contenido de humedad del producto (Muños et al., 2014).

Para la variable dureza a nivel sensorial no se presentaron diferencias estadísticas significativas con un valor $p>0,05$; la dureza en el patrón fue de 2,89 y se encontraron muestras con menor dureza $(2,4)$ como las aplicaciones de MF 3, 8, 10, 13 y 14; a su vez la muestra 1 fue la calificada con un valor más alto (3,21); a nivel instrumental las muestras que presentaron diferencias estadísticas significativas con un valor de $\mathrm{p}<0,05$ fueron el patrón con los tratamientos $3,6,10,11,13$ y 14; se puedo evidenciar que algunos tratamientos presentaron el mismo comportamiento tanto a nivel sensorial como instrumental, el patrón presentó un valor promedio de 7747,97 gramos fuerza; se observa un grupo homogéneo conformado por las muestras 4, 8, 1, 5, 17, 18, 2, 15, 16, 12 y el patrón; la muestra 7 ( 5331,7 gf) presentó el menor valor de dureza. En la Fig. 5a se muestra el comparativo a nivel instrumental y sensorial, se presentan dos comportamientos de estas variables con las metodologías utilizadas; el primero asociado a la capacidad de formación de gel de cada una de las mezclas dentro del producto cárnico, lo que hace incrementar o disminuir estos valores, y el segundo asociado con la CRA de cada una de las MF.
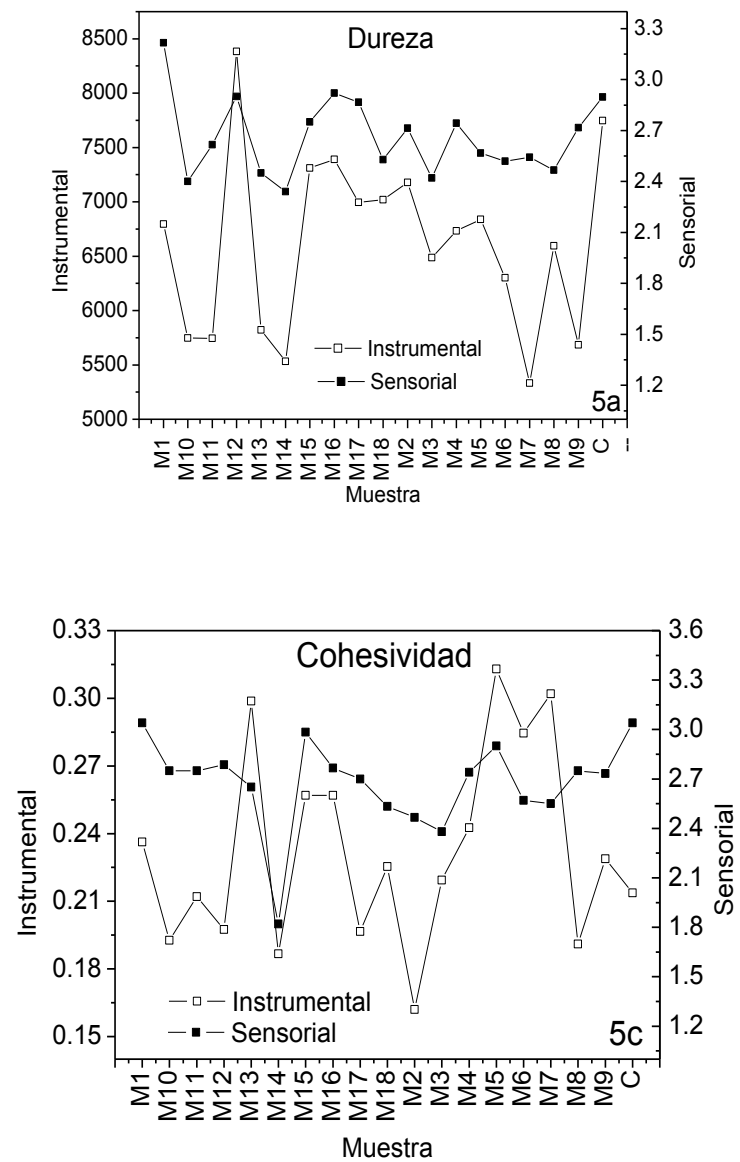
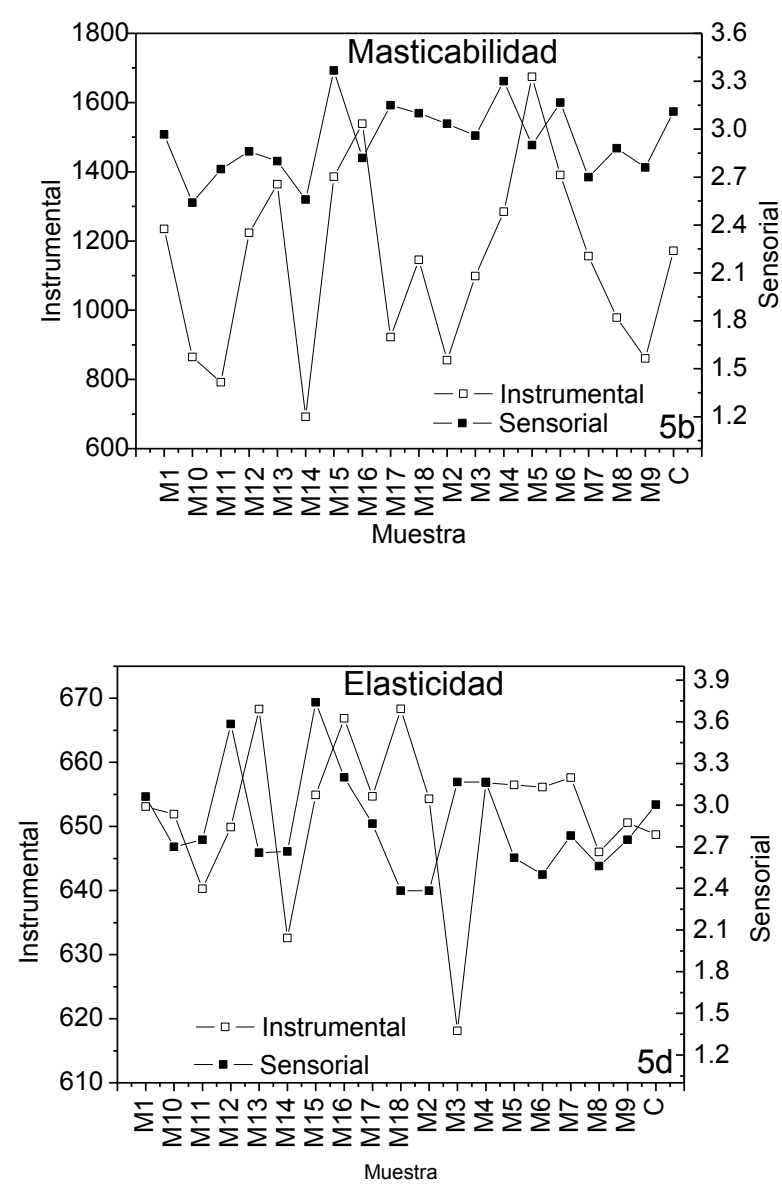

Fig. 5: Correlación de algunos parámetros sensoriales evaluados por técnicas instrumentales y sensoriales 
La cohesividad del producto cárnico a nivel sensorial presentó diferencias estadísticamente significativas con un valor $p<0,05$; entre el patrón y las muestras $2,3,6,7,14,18$, se encontró que el patrón fue más cohesivo que los tratamientos exhibiendo un valor de 3,04; la mezcla menos cohesiva fue la 14 con un valor de 1,82; las muestras 1 y 15 presentaron una cohesividad similar al patrón, para las otras mezclas esta característica oscilo entre 2,38 y 2,53; se observó un grupo homogéneo entre el patrón y las mezclas: 13 , $17,9,4,11,10,8,16,12,5$ y 15 . A nivel instrumental se presentaron diferencias estadísticas significativas con un valor $p<0,05$ entre el patrón y las muestras $13,5,6$, y 7 ; y formando un grupo homogéneo las muestras 2, 14, 8,10,17,12,11, patrón, $3,18,9$, y 1; el patrón presentó un valor de 0,213 ; mientras que la mezcla 5 tuvo el mayor valor de cohesividad de 0,31, y el más bajo para la mezcla 2, con un valor de 0,16, en la Fig. 5b, se presenta el comportamiento de esta variable.

Para la elasticidad a nivel sensorial se presentaron diferencias estadísticamente significativas entre el patrón y las muestras 2, 15, 18; las muestras 2 y 18 tuvieron una calificación de 2,38; mientras que el patrón tuvo una mayor elasticidad $(3,00)$ por otro lado la muestra 15 resulto más elástica con un valor de 3,74 para este parámetro evaluado en una escala de 0-7. A nivel instrumental se presentaron diferencias estadísticas significativas entre el patrón y las muestras $3,13,16$ y 18 ; formaron un grupo homogéneo las muestras 11 , $8,12,9,10,1,2,17,15,6,5,4,7$ y patrón. Según las metodologías empleadas en la evaluación de esta variable no hubo correlación en los resultados obtenidos por los métodos de evaluación aplicados como se visualiza en la Fig. 5c.

A nivel sensorial la masticabilidad de las aplicaciones de $M F$ tuvo un valor promedio de 3,11 ; entre el patrón las mezclas 5,7 y 17 no se presentaron diferencias estadísticas significativas con un valor $p>0,05$, en las Fig. $5 \mathrm{~d}$ se presentan los comportamientos a nivel sensorial e instrumental. Se detectaron dos grupos homogéneos: el primero conformado por las muestras 10 y 14 cuya masticabilidad fue de 2,5 y el grupo restante está conformado por las otras mezclas incluyendo el patrón cuyo valor de masticabilidad fue de 3,11; a nivel instrumental se presentó diferencias estadísticas entre el patrón y las muestras 11, 14, y 5, el patrón exhibió un valor de 1171,53; formando un grupo homogéneo entre las muestras 16, 6, 15, 13, 4, 1, 12 7, 18, 3 y patrón. Reportando el menor valor la aplicación de MF 14 y el valor más alto por la mezcla 5.

Las variables sabor extraño, dureza y masticabilidad sensorial, no presentaron diferencias significativas para las diferentes muestras, siendo esto positivo ya que son variables determinantes en la calidad sensorial del producto terminado. Se evidenció una buena correlación en dureza (5a) y masticabilidad (5b) evaluada por ambos métodos lo que sugiere una buena calibración del panel sensorial. Con base a los resultados encontrados a nivel sensorial e instrumental se seleccionaron las muestras 5, 7 y 17 al tener una mayor similitud frente al patrón en los parámetros evaluados por las dos técnicas aplicadas.

\section{CONCLUSIONES}

Con base a los resultados encontrados y después de realizar su discusión y análisis, se pueden obtener las siguientes conclusiones sobre la extracción de fibra de naranja y su aplicación en productos cárnicos emulsionados tipo salchicha: 1) Se eliminaron sabores amargos y cítricos en la cáscara de naranja, con lo cual se puede incluir en productos cárnicos hasta un nivel del 2,5\%(w/w). 2) Se desarrollaron nuevas metodologías para la producción y caracterización de derivados cárnicos a escala piloto; lo cual representa ahorros en tiempo y costo en el desarrollo y formulación de nuevos productos para esta industria. 3) Fueron seleccionadas las mezclas 5,7 , y 17 que presentaron mayor similitud a la muestra control en términos de dureza, cohesividad, elasticidad, jugosidad, pudiendo reemplazar hasta un $50 \%$ de grasa en la formulación 4) Se establecieron metodologías reológicas para evaluar la incidencia del tratamiento térmico sobre la estructuración (gelificación) de la pasta cárnica y sobre las propiedades viscoelásticas de las mezclas de fibras y almidones en agua.

\section{AGRADECIMIENTOS}

A Colciencias por permitir la financiación esta investigación en torno al desarrollo de mezclas de fibras y almidones como reemplazante de grasa en la modalidad de incentivo tributario código 338756236197, a la Fundación Intal, a TECNAS S.A y a todos los participantes en la ejecución y desarrollo de esta propuesta.

\section{REFERENCIAS}

Alarcón, M. A., López, J. H., y Restrepo, D. A. Caracterización de la Funcionalidad Tecnológica de una Fuente Rica en Fibra Dietaría Obtenida a partir de Cáscara de Plátano. Rev. Fac. Nal. Agr. Medellín, 66 (1), 6959-6968 (2013) 
AOAC. 923-03 -1923. Association of Official Analytical Chemists, Official Methods of Analysis. Ash of flour, Direct method. Washington http://www.aoacofficialmethod.org (2012)

AOAC. 988.05A. Association of Official Analytical Chemists. Official Methods. Of Analysis. Protein (crude) in animal feed. CuSO4/TiO2 mi. Washington http://www.aoacofficialmethod.org (1990)

AOAC. 934.01. Association of Official Analytical Chemists. Official Methods of Analysis. Loss on drying (Moisture) at $95^{\circ}-100$. Washington. http://www.aoacofficialmethod.org (2012)

AOAC. 993.19-1996. Association of Official Analytical Chemists. Official Methods of Analysis. Soluble dietary fiber in food and food. Washington http://www.aoacofficialmethod.org (2000)

AOAC. 991.42--1994. Association of Official Analytical Chemists. Official Methods of Analysis. Insoluble dietary fiber in foods. Washington http://www.aoacofficialmethod.org (1994)

Akesowan, A., Effect of soy protein isolate on quality of light pork sausages containing konjac flour. 7(24), 4586-4590 (2008)

Bloukas, J., Paneras, E., \& Papadima, S. Effect of carrageenan on processing and quality characteristics of low-fat frankfurters. Journal of Muscle Foods, 8 (1) 63-83 (1997)

Cañas Á., Z. Aplicación de concentrado de cáscara de arveja (pisum sativum) como fuente de fibra dietaria en derivado cárnico (hamburguesa). Univeridad Nacional de Colombia, Facultad de Ciencias Agropecuarias (2010)

Cañas Á., Z., Molina R., D. A., \& Cortes R., M. Revisión: Productos Vegetales como Fuente de Fibra dietaría en la Industria de Alimentos. Revista Facultad Nacional de Agronomía, Medellín, 64(1), 6023-6035 (2011)

Delgado, N., y Albarracín, W. Microestructura y propiedades funcionales de harinas de quinua (Chenopodioum quinoa w) y chachafruto (Erythrina edulis): Potenciales extensores cárnicos. Vitae, 19(1), 430-432 (2012)

Estadísticas de producción nacional, Agronet (visitada 10 Enero de 2015). www.agronet.gov.co

García, M. L., Cáceres, E., \& Selgas, M. D. Utilisation of fruit fibres in conventional and reduced-fat cookedmeat sausages. Journal of the Science of Food and Agriculture, 87, 624-631 (2007)

Grigelmo - Miguel, N., y Martõân-belloso, O. Characterization of dietary fi ber from orange juice extraction. Food Research International, 31(5), 355-361(1999)

León, Liliana Serna Cock, C. T. Potencial agroindustrial de cáscaras de mango (Mangifera indica variedades Keitt y Tommy Atkins. 64(2) 2223-0118 (2014)

Meilgard. M, Civille. G, Carr T. Sensory Evaluation Techniques. CRC Press LLC. $3^{\text {rd }}$ Edition. United States of America. p. 195-197. (1999)

Meneses, S. M. O., Molina, D. A. R., y Vargas, J. H. L. Derivados cárnicos como alimentos funcionales. Revista Lasallista de Investigacion, 8(2), 163-172 (2011)

Muños, S. A., Restrepo, D. A., y López J. H. Efecto de la Inclusión de Inulina en Salmueras de Marinado sobre Mermas y Calidad Sensorial de Pechugas de Pollo,. Rev. Fac. Nal. Agr. Medellín, 67(45), 7219-7228 (2014)

Ministerio de la Protección Social de Colombia, Resolución 333 del 2008, Bogotá, Colombia

NTC 4489, Instituto Colombiano de Normas Técnicas, Bogotá, Colombia, (1998)

NTC 4129, Instituto Colombiano de Normas Técnicas, Bogotá, Colombia, (1998)

Pons, M., y Fiszman, S. M. Instrumental Texture Profile Analysis With Particular Reference To Gelled Systems. Journal of Texture Studies, 27(6), 597-624 (1996) 
Restrepo Duque, A. M., Rodríguez Sandoval, E., y Manjarrés Pinzón, K. Cortezas de naranja comestible: una aproximación al desarrollo de productos con valor agregado a partir de residuos agroidustriales. Rev.

Producción + Limpia, 6(2), 47-57 (2011)

Simplicio de Santana, M. de F. Caracterização físico-química de fibra alimentar de laranja e maracujá. Campinas (2005)

Tokusoglu, Ö. y Ünal, M. K. Fat Replacers in Meat Products. Pakistan Journal of Nutrition, 2(3), 196-203 (2003)

Verma, A. K., y Banerjee, R. Dietary fiber as functional ingredient in meat products: a novel approach for healthy living - a review. Journal of Food Science and Technology, 47(3), 247-57 (2010) 